\title{
Exploration of CdTe quantum dots as mesoscale pressure sensors via time-resolved shock- compression...
}

Article in Journal of Applied Physics · July 2016

DOI: $10.1063 / 1.4959257$

CITATIONS

0

11 authors, including:

\section{David Scripka}

Georgia Institute of Technology

6 PUBLICATIONS 4 CITATIONS

SEE PROFILE

\section{Dana D Dlott}

University of Illinois, Urbana-Champaign 334 PUBLICATIONS 7,922 CITATIONS

SEE PROFILE
READS

33

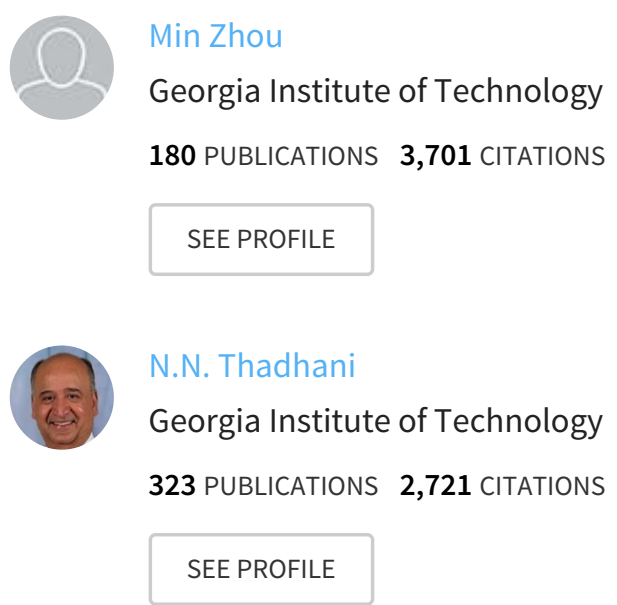

Some of the authors of this publication are also working on these related projects: 


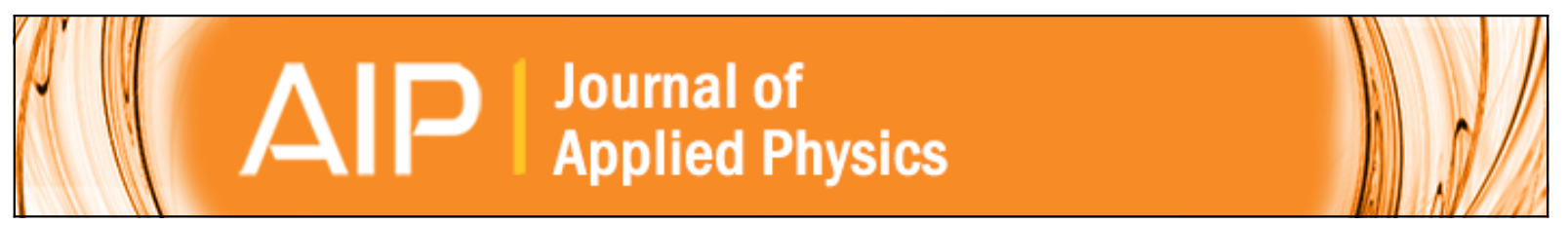

Exploration of CdTe quantum dots as mesoscale pressure sensors via time-resolved shock-compression photoluminescent emission spectroscopy

Zhitao Kang, Alexandr A. Banishev, Gyuhyon Lee, David A. Scripka, Jennifer Breidenich, Pan Xiao, James

Christensen, Min Zhou, Christopher J. Summers, Dana D. Dlott, and Naresh N. Thadhani

Citation: Journal of Applied Physics 120, 043107 (2016); doi: 10.1063/1.4959257

View online: http://dx.doi.org/10.1063/1.4959257

View Table of Contents: http://scitation.aip.org/content/aip/journal/jap/120/4?ver=pdfcov

Published by the AIP Publishing

\section{Articles you may be interested in}

Temperature-sensitive photoluminescent CdSe-ZnS polymer composite film for lock-in photothermal characterization

J. Appl. Phys. 119, 224902 (2016); 10.1063/1.4953591

Laser-excited optical emission response of CdTe quantum dot/polymer nanocomposite under shock compression

Appl. Phys. Lett. 108, 011908 (2016); 10.1063/1.4939701

Ligand exchange leads to efficient triplet energy transfer to CdSe/ZnS Q-dots in a poly(N-vinylcarbazole) matrix nanocomposite

J. Appl. Phys. 113, 083507 (2013); 10.1063/1.4793266

CdSe quantum dots-poly(3-hexylthiophene) nanocomposite sensors for selective chloroform vapor detection at room temperature

Appl. Phys. Lett. 101, 173108 (2012); 10.1063/1.4762861

Localization and interdot carrier transfer in CdSe and CdZnMnSe quantum dots determined by cw and timeresolved photoluminescence

Appl. Phys. Lett. 90, 201916 (2007); 10.1063/1.2740484

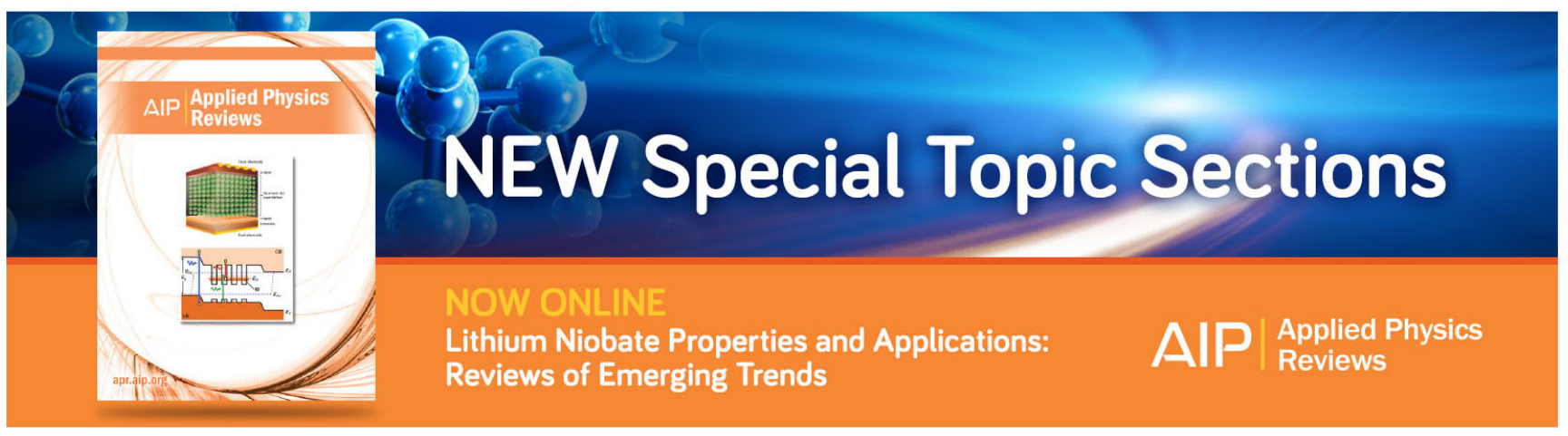




\title{
Exploration of CdTe quantum dots as mesoscale pressure sensors via time-resolved shock-compression photoluminescent emission spectroscopy
}

\author{
Zhitao Kang, ${ }^{1,2}$ Alexandr A. Banishev, ${ }^{3}$ Gyuhyon Lee, ${ }^{2}$ David A. Scripka, ${ }^{2}$ \\ Jennifer Breidenich, ${ }^{2}$ Pan Xiao, ${ }^{4,5}$ James Christensen, ${ }^{3}$ Min Zhou, ${ }^{5}$ \\ Christopher J. Summers, ${ }^{2}$ Dana D. Dlott, ${ }^{3}$ and Naresh N. Thadhani ${ }^{2, a)}$ \\ ${ }^{1}$ Georgia Tech Research Institute, Georgia Institute of Technology, Atlanta, Georgia 30332-0826, USA \\ ${ }^{2}$ School of Materials Science and Engineering, Georgia Institute of Technology, Atlanta, Georgia 30332-0245, USA \\ ${ }^{3}$ School of Chemical Sciences and Fredrick Seitz Materials Research Laboratory, University of Illinois \\ at Urbana-Champaign, Urbana, Illinois 61801, USA \\ ${ }^{4}$ LNM, Institute of Mechanics, Chinese Academy of Sciences, Beijing 100190, China \\ ${ }^{5}$ George W. Woodruff School of Mechanical Engineering, Georgia Institute of Technology, Atlanta, \\ Georgia 30332-0405, USA
}

(Received 18 March 2016; accepted 11 July 2016; published online 28 July 2016)

\begin{abstract}
The nanometer size of CdTe quantum dots (QDs) and their unique optical properties, including size-tunable narrow photoluminescent emission, broad absorption, fast photoluminescence decay, and negligible light scattering, are ideal features for spectrally tagging the shock response of localized regions in highly heterogeneous materials such as particulate media. In this work, the timeresolved laser-excited photoluminescence response of QDs to shock-compression was investigated to explore their utilization as mesoscale sensors for pressure measurements and in situ diagnostics during shock loading experiments. Laser-driven shock-compression experiments with steady-state shock pressures ranging from 2.0 to $13 \mathrm{GPa}$ were performed on nanocomposite films of CdTe QDs dispersed in a soft polyvinyl alcohol polymer matrix and in a hard inorganic sodium silicate glass matrix. Time-resolved photoluminescent emission spectroscopy was used to correlate photoluminescence changes with the history of shock pressure and the dynamics of the matrix material surrounding the QDs. The results revealed pressure-induced blueshifts in emitted wavelength, decreases in photoluminescent emission intensity, reductions in peak width, and matrix-dependent response times. Data obtained for these QD response characteristics serve as indicators for their use as possible time-resolved diagnostics of the dynamic shock-compression response of matrix materials in which such QDs are embedded as in situ sensors. Published by AIP Publishing.
\end{abstract}

[http://dx.doi.org/10.1063/1.4959257]

\section{INTRODUCTION}

The shock-compression behavior of highly heterogeneous materials, such as particulate media, is dominated by mesoscale effects arising from collapse of voids, as well as shock wave interactions with heterogeneities (e.g., particle boundaries and interfaces between constituents with dissimilar material properties). For applications involving materials subjected to shock-compression or dynamic high strain rate loading, understanding the influence of mesoscale heterogeneities on the bulk behavior/response of such materials is of paramount importance. There is, therefore, a significant need for diagnostic methods and sensor materials that can provide direct in situ measurements of pressure (and other parameters) with appropriate time- and spatial resolutions relevant to the understanding of the dynamics of shock compression processes in inert and reactive particulate materials. The limitations of shock-compression experiments in providing the mesoscopic details of deformation and mass flow in particulate materials have long been recognized. ${ }^{1}$ Conventional diagnostics, such as in situ piezoresistive/piezoelectric stress gauges, ${ }^{2,3}$ provide time resolutions down to the nanosecond

\footnotetext{
a) Author to whom correspondence should be addressed. Electronic mail: naresh.thadhani@mse.gatech.edu
}

scale but have spatial resolutions spanning the area of the sensor element of several millimeters. The current state-ofthe-art in velocity interferometry, namely, "line VISAR" or "ORVIS" (Optically recording velocity interferometer system), allows for the collection of velocity profiles along a line ${ }^{4}$ providing information inherent to the heterogeneous effects along the linear dimension (typically up to couple of millimeters); however, it remains a surface-sensitive diagnostic method that averages the effects of the volume of the material being diagnosed.

Mesoscale sensing in shock-compression experiments requires the sensor material to be small in size and possess pressure-dependent physical properties which can be captured and recorded at the nanosecond scale to achieve high temporal resolution. ${ }^{5,6}$ Quantum dots (QDs) are semiconducting nanoparticles of a few to tens of nanometers. These robust inorganic photoemitters exhibit size-dependent emission spectra in the whole visible and near-infrared range, due to quantum confinement effects. ${ }^{7}$ Heterogeneous materials consisting of different constituents can have each component tagged with different-sized QDs. The QDs can be excited with a single wavelength, while the photoluminescent emission can consist of multiple discrete narrowband components corresponding to each tagged constituent, thereby providing 
a great advantage for multiplexed sensing at different locations or different interfaces within the heterogeneous sample. The photoluminescence decay time of QDs is in the picosecond to nanosecond range; thus, a fast temporal response can be achieved. Nanoparticles that are small compared to optical wavelengths exhibit negligible light scattering when incorporated into transparent matrices. The optical properties of QDs have been reported to be pressure- and straindependent as a result of changes in their bandgap with particle size. ${ }^{8,9}$ Therefore, QDs can potentially be used as sensor materials for investigating the time-resolved mesoscale effects of shock-compression of highly heterogeneous materials, including compacts of inert or reactive powders, in order to obtain unprecedented information about local particle or ensemble behavior, as well as the dynamics of the surrounding materials during high-strain-rate or shockcompression loading.

Hydrostatic compression of QDs has been observed to produce photoluminescent emission blueshifts that increase monotonically with pressure. ${ }^{10-12}$ In our recent theoretical study ${ }^{13}$ on QDs subjected to shock-compression and associated non-hydrostatic loads, pressure-induced blueshifts were observed to initially increase to a maximum and then decrease. The effect was also confirmed through experimental studies ${ }^{13}$ of the photoluminescent emission spectra of shocked $\sim 3 \mathrm{~nm}$ CdTe QDs in a hydrophilic polymer matrix (poly-vinyl alcohol; PVA). However, the time response of the measured blueshift from the QDs in the PVA matrix was not very fast (of the order of $\sim 60 \mathrm{~ns}$ ), indicating that the highly sluggish mechanics of the surrounding (polymer matrix) medium plays an important role in influencing the optomechanical response of the QDs. Therefore, the effect of embedding the QDs in different matrix media, on the time resolution of the QD response, needs to be studied. In the present work, we have investigated and compared the shockcompression response of CdTe QDs dispersed in both a soft PVA polymer matrix and a hard sodium silicate glass matrix. Studies of these two types of matrix media, hereafter referred to as "polymer" and "glass," respectively, are also relevant for the understanding of the response of two widely-used embedded shock sensors, namely, the polyvinylidene fluoride polymer (PVDF) ${ }^{14}$ and quartz stress gauges. ${ }^{15}$

In addition to matrix effects, many other issues need to be addressed and understood prior to the possible use of QDs as pressure sensors for in situ probing of the shockcompression response of heterogeneous materials. The QD structure and surface chemistry need to be tuned to maintain compatibility with the media of interest. Previous studies have indicated that positively-charged QDs are needed with the PVA matrix, since negatively-charged QDs heavily aggregate in PVA, leading to a complex and abnormal spectroscopic response. As shown in our previous theoretical study, ${ }^{13}$ the overall shock-induced blueshift mechanism can be understood based on strain tuning of the QD bandgap using a simplified model of a QD without surface capping groups in a matrix. However, factors such as shock-induced photoluminescent emission intensity change, peak width change, and the possibility of dynamic phase transformations, require more detailed experimental studies. In the present work, the response of CdTe QDs of different sizes and capping groups dispersed in a polymer or glass (matrix) media was investigated to understand the effects of dynamic high-pressure (shock-compression) loading on their photoluminescence spectral response.

\section{SYNTHESIS OF CdTe QDS AND NANOCOMPOSITE FILMS}

Colloidal CdTe QDs were synthesized by adding freshly prepared NaHTe solution to $\mathrm{N}_{2}$-saturated $\mathrm{Cd}\left(\mathrm{ClO}_{4}\right)_{2}$ solutions in the presence of 2-mercaptoethylamine (MEA) or 3-mercaptopropionic acid (MPA) as the stabilizing agents and water as the solvent. The solutions were then heated to the boiling temperature and refluxed for different times to grow and obtain QDs of controlled particle sizes. The detailed synthesis procedure is reported elsewhere. ${ }^{16,17}$ The surface chemistries of the QDs were altered to prepare nonaggregated nanocomposite films. For incorporation in the PVA matrix, we used MEA-capped QDs, which had net positive charge due to $-\mathrm{NH}_{4}^{+}$(ammonium) capping groups. For incorporation in the glass matrix, a liquid sodium silicate solution (obtained from Sigma-Aldrich) with MPA-capped QDs, which had net negative charge due to the $-\mathrm{COO}^{-}$ (carboxylate) end groups, was used.

The MEA-capped QDs in the nanocomposite polymer films were $\sim 3 \mathrm{~nm}$ in size and emit at a characteristic wavelength of $586 \mathrm{~nm}$. The MPA-capped QDs in the nanocomposite glass films were $\sim 4 \mathrm{~nm}$ in size and emit at a characteristic wavelength of $674 \mathrm{~nm}$. A dual-emitting composite film sample with a PVA matrix was also prepared using both $582 \mathrm{~nm}$ and $632 \mathrm{~nm}$ emitting MEA-capped QDs. After the QDs were dispersed at room temperature in the respective polymer, or glass (water-based sodium silicate solution), matrix to form solutions of appropriate concentration and viscosity, nanocomposite thin film samples were prepared by drop-coating onto $25.4 \times 25.4 \times 0.5 \mathrm{~mm}^{3}$ glass substrates (Delta Technologies) followed by air drying to form homogeneous non-aggregated $\sim 30 \mu$ m thick films with $\sim 0.15$ wt. \% QD concentration.

\section{SETUP OF LASER-DRIVEN SHOCK-COMPRESSION EXPERIMENTS}

The shock-compression experiments involved a laserlaunched Al-flyer plate impacting on QD-containing nanocomposite films, using the setup developed by the Dlott group. ${ }^{18} \mathrm{~A}$ schematic illustration of the experimental set-up is shown in Figure 1, with details described previously. ${ }^{18}$ Photon Doppler velocimetry (PDV) ${ }^{19}$ was used to monitor the flyer plate velocity history via a laser beam that transmitted through the transparent sample and reflected back into a detector from the flyer surface, while simultaneously, time-resolved spectroscopic photoluminescence spectra were obtained from the laserexcited QDs. The flyer plates were launched from an assembly consisting of a glass substrate $6.35 \mathrm{~mm}$ in thickness and an aluminum foil (Alufoil) $5 \times 5 \mathrm{~cm}^{2}$ in area and $50 \mu \mathrm{m}$ in thickness which were bonded together using low-viscosity Eccobond 24 water-clear epoxy (Henkel Loctite). A spatially homogenized Q-switched Nd:YAG laser (Quanta-Ray Pro-350) was 


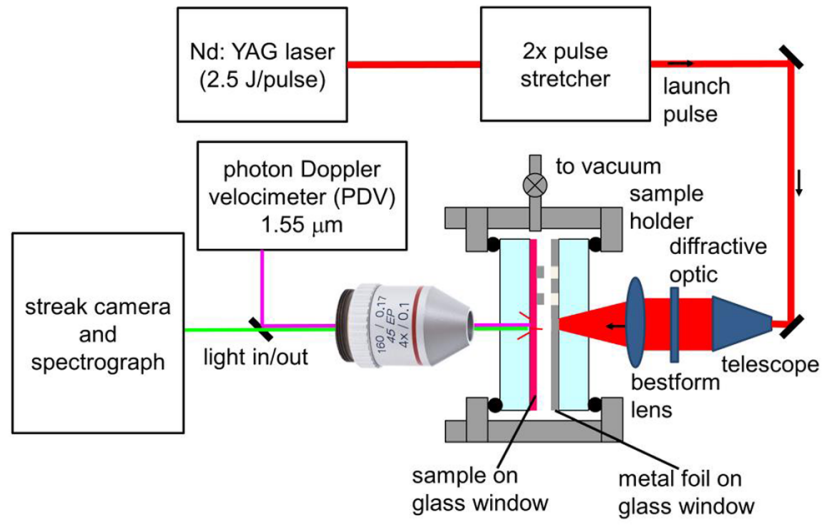

FIG. 1. Experimental setup for laser-driven flyer impact experiments for shock-compression of CdTe QDs dispersed in a polymer or glass matrix.

used to launch the flyer, producing shock pulses of $\sim 20 \mathrm{~ns}$ in duration following the impact on the sample. The flyer plate velocities measured with PDV were controlled by varying the launch pulse energy up to a maximum of $2.5 \mathrm{~J} /$ pulse. The laser launch beam has a central $0.5 \mathrm{~mm}$ diameter region whose intensity is uniform to within $\pm 2 \%$. An all-reflective beam stretcher was used to stretch the nominal $10 \mathrm{~ns}$ laser pulse to $20 \mathrm{~ns}$ full-width half-maximum. ${ }^{20}$ The PDV and spectroscopic measurements probed smaller regions of $0.07 \mathrm{~mm}$ and $0.2 \mathrm{~mm}$ in diameter, respectively, at the center of the uniform launch pulse. ${ }^{18}$ The QD photoluminescent emission was excited by $250 \mathrm{~ns}$ FWHM pulses from a Q-switched $527 \mathrm{~nm} \mathrm{Nd:YLF}$ laser (Quantronix $527 \mathrm{DP}$ ) and recorded with $0.5 \mathrm{~ns}$ effective time per CCD pixel using a streak camera. Displayed data show each row of the streak camera out spaced at $0.5 \mathrm{~ns}$ intervals. The overall time resolution of the system was 2 ns.

The PDV measurements probed the velocity history of the impact surface of the $50 \mu \mathrm{m}$ thick Al flyer plates. After the initial acceleration transient, the flyer plate travels freely (in vacuum) at a velocity denoted as $U_{f}$. When the plates impact the targets, the velocity drops to a value denoted as $U_{p}$ which is the velocity of the flyer/target interface, or alternatively the material velocity of the shocked target at the impact face. The measured values of $U_{p}$ averaged over the time interval of the steadily-driven shocks and averaged over ten shock events, typically varied by $\pm 2 \%$ (one standard deviation). The calculated pressure uncertainty then depends on the Hugoniot for each medium, and a reasonable estimate is $\pm 5 \%$.

\section{RESULTS AND DISCUSSIONS}

Two examples of time-stream photoluminescent emission spectra of samples impacted by $50 \mu \mathrm{m}$ thick flyers, recorded by a spectrograph and streak camera, are shown in Figure 2, with one time streak for $586 \mathrm{~nm}$ emitting CdTe QDs in polymer (with $U_{f}=1.12 \mathrm{~km} / \mathrm{s}$ ) and the other for $674 \mathrm{~nm}$ emitting CdTe QDs in glass with $U_{f}=0.89 \mathrm{~km} / \mathrm{s}$. Figures 2(a) and 2(b) present the data as contour plots of the streak camera output, and Figures 2(c) and 2(d) display the corresponding photoluminescent emission spectra. The QDs in both samples exhibit shock-induced blueshift and intensity loss. The CdTe QDs are direct band gap semiconductors with strong quantum confinement effects, and shockcompression leads to increases in band gap energies and corresponding blueshifts in photoluminescent emission wavelength. ${ }^{10,12}$ Note that the shock response of the QDs, measured in terms of the time required for attaining the peak wavelength shifts and intensity changes, is much faster in glass than in the polymer.

The photoluminescent emission spectra were processed to extract time-dependent intensity changes, wavelength blueshifts, and spectral (or peak) widths, using the method of moments proposed by Brown et al., ${ }^{18}$ which is convenient (a)

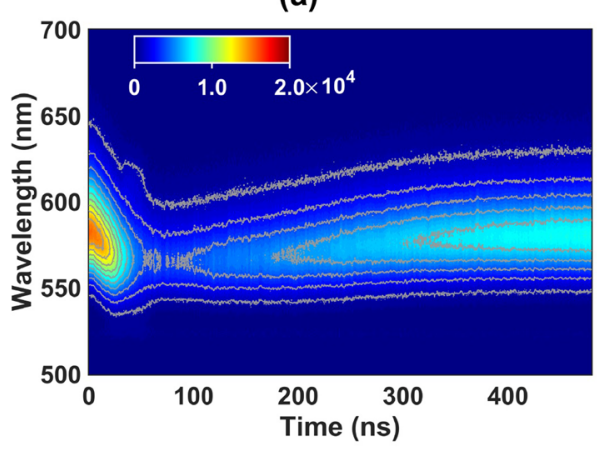

(c)

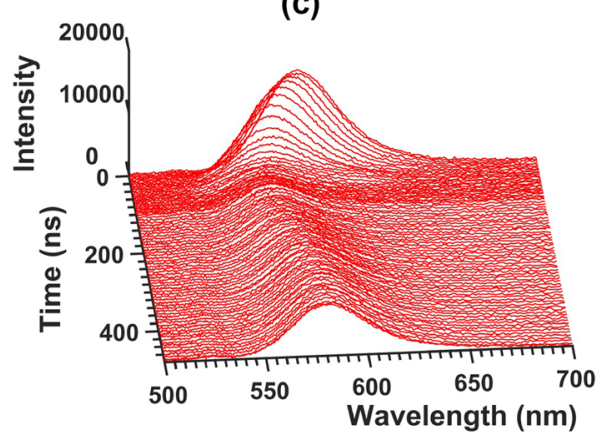

(b)

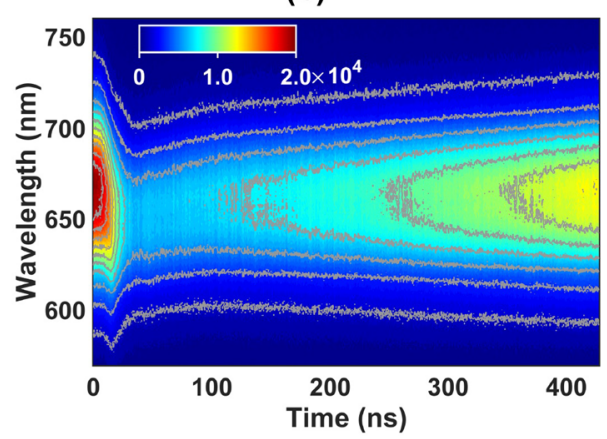

(d)

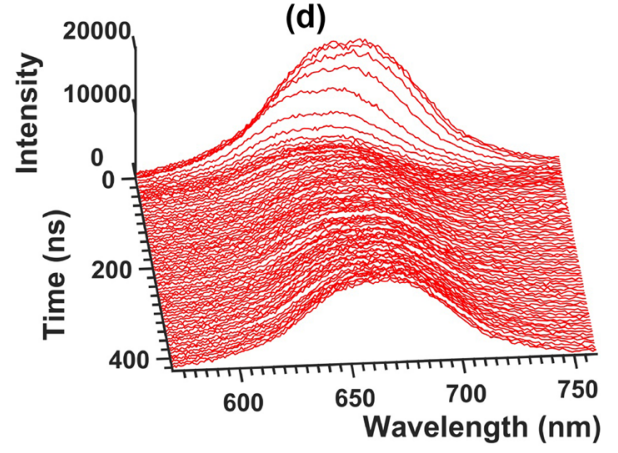

FIG. 2. Time-resolved photoluminescent emission spectra for QDs in polymer and glass matrix with shocks generated by impact at time $t=0$ by $50 \mu \mathrm{m}$ thick Al flyers. (a) Contour plot and (c) corresponding emission spectra for $586 \mathrm{~nm}$ emitting QDs in PVA polymer with impact velocity $U_{f}=1.12 \mathrm{~km} / \mathrm{s}$. (b) Contour plot and (d) emission spectra for $674 \mathrm{~nm}$ emitting QDs in glass with impact velocity $U_{f}=0.89 \mathrm{~km} / \mathrm{s}$. The QD emitters show shock-induced blueshift and intensity loss. 
(a)

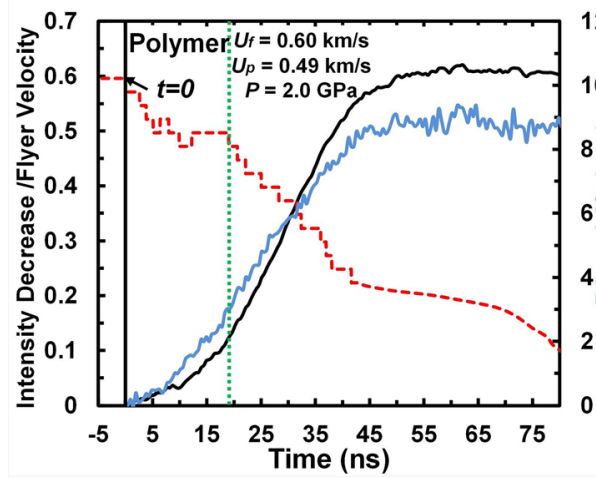

(b)

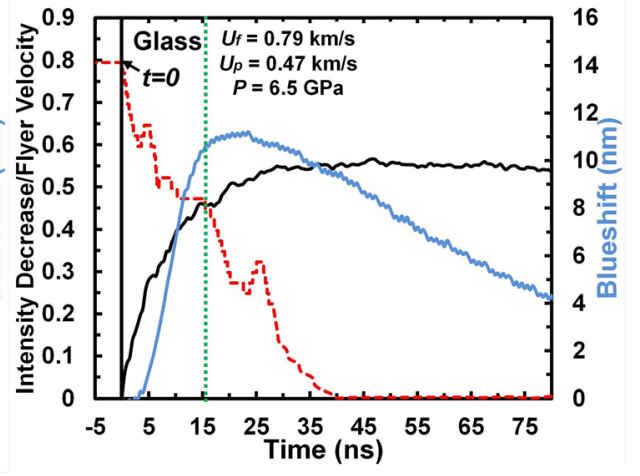

FIG. 3. The segmented red curves denote the flyer plate velocity profiles and the dotted green lines denote the end of the steadily-driven shocks. The solid black and blue curves denote the emission intensity decrease and blueshift, respectively. The values of $U_{p}$, the flyer/sample interface velocity, are almost the same in both experiments. Due to the higher impedance of glass, the free-surface flyer velocity $U_{f}$ has to be greater to produce the same $U_{p}$. At the same $U_{p}$, the pressure in glass is much greater. The shock-induced blueshift and intensity loss are close to their maximum values at the end of the shock in glass, but a much slower response is observed in the polymer.

for automated analysis of time-streams of spectra. The zeroth moment is the wavelength-integrated photoluminescent emission intensity, the first moment is the average wavelength blueshift, and the square-root of the second moment is the spectral width. The effects of the polymer or glass matrix are presented and discussed in Sections IV A-IV E.

\section{A. Effect of matrix material on response time}

Figure 3 shows typical plots of flyer velocity profiles, fractional photoluminescent emission intensity decreases, and blueshifts for QDs in the respective polymer and glass matrices. The initial part of the flyer velocity profiles (dashed red curves) shows the flyer approaching the sample at $U_{f}=0.6 \mathrm{~km} / \mathrm{s}$ in the polymer case and at $U_{f}=0.79 \mathrm{~km} / \mathrm{s}$ in the glass case. Upon impact at $t=0$, the velocities drop to the level denoted $U_{p}$ over a time interval denoted $\tau_{\text {drop }} . U_{p}$ denotes the velocity of the flyer/sample interface. The value of $\tau_{\text {drop }}$, typically a few nanoseconds, arises from the imperfect planar contact process due to the curvature and roughness on the order of microns of the flyer and target surface pair. Despite the different values of $U_{f}$ shown in Figures 3(a) and $3(\mathrm{~b})$, the values of $U_{p}$ for the polymer and glass samples are nearly identical $(0.49 \mathrm{~km} / \mathrm{s}$ and $0.47 \mathrm{~km} / \mathrm{s})$, due to the differing shock impedances of the samples. The value of $U_{p}$ remain steady for a time interval denoted $\tau_{p 1}$ before decaying gradually to zero over several tens of nanoseconds. In the experiments reported here, $\tau_{p l}=13.9 \mathrm{~ns}$, and it represents the duration of steady-state shock drive in the samples.

The value of the steady-state pressure in the material can be determined from the measured value of $U_{p}$ and tabulated properties ${ }^{18,21}$ of the polymer and glass via the shock and particle velocity empirical relationship $U_{s}=a+b \cdot U_{p}$ and the Rankine-Hugoniot jump condition

$$
P-P_{0}=\rho_{0} U_{s} U_{p},
$$

where $U_{s}, \rho_{0}$, and $P$ are the shock velocity, initial density, and pressure, respectively. Note that the Hugoniot pressure, $P$, is the steady-state stress, although it will be referred synonymously as pressure throughout the paper. For the PVA polymer, $a=2.46 \mathrm{~km} / \mathrm{s}$ and $b=1.565 .^{21}$ Corresponding parameters for the liquid-deposited sodium silicate glass were not available, so we estimated the glass pressures based on parameters for a related soda-lime glass. ${ }^{22}$

The calculated pressure in the polymer sample in Figure 3 (a) is $2.0( \pm 0.1) \mathrm{GPa}$, and in the glass sample in Figure 3 (b) it is $6.5( \pm 0.2) \mathrm{GPa}$. Since the QD-doped glass was on a closely impedance-matched glass substrate, shock reflections were minimal so the sample was subjected to a single-stage shock. The PVA has acoustic impedance lower than glass. When shock waves propagate from PVA to glass, there is a shock reflection back into PVA. Therefore, in PVA we have both direct and reflected waves and the pressure rises in the PVA as a two-stage process.

The end of the steady shock drive in Figure 3, at 19 ns for the polymer and $15.5 \mathrm{~ns}$ for the glass, is indicated by vertical green lines. With the glass sample (Figure 3(b)), the blueshift and intensity loss are close to their maximum values at the end of the shock drive, but with the polymer sample, the blueshift and intensity loss are just beginning, and these parameters continue to increase significantly until about $60 \mathrm{~ns}$. The slower time response of the polymer is possibly due to its sluggish viscoelastic shock-compression behavior. $^{23}$ The glass response is much faster because glasses are elastic below the Hugoniot elastic limit (HEL, reported to be $\sim 3.5 \mathrm{GPa}$ by Alexander and co-workers ${ }^{22}$ ), and approximately fluid above this limit. ${ }^{24}$

\section{B. Shock-induced spectral blueshift}

Time-dependent QD spectral blueshifts at a series of pressures are shown in Figures 4(a) and 4(b) for polymerand glass-matrix samples. The values of the blueshifts at the end of the steadily-driven shocks (defined as the blueshift at $t=20 \mathrm{~ns}$ for simplicity) and the maximum blueshift values are shown in Figures 4(c) and 4(d). Due to the slower response in polymer, the blueshift at $20 \mathrm{~ns}$ is noticeably smaller than the maximum value. In the faster-responding glass, the blueshift at $20 \mathrm{~ns}$ is already close to its maximum value. 
(a)

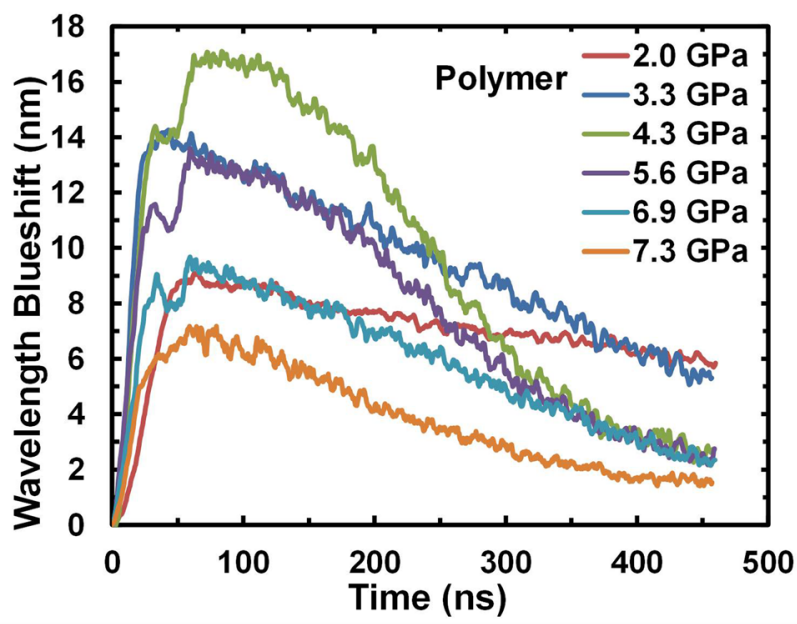

(c)

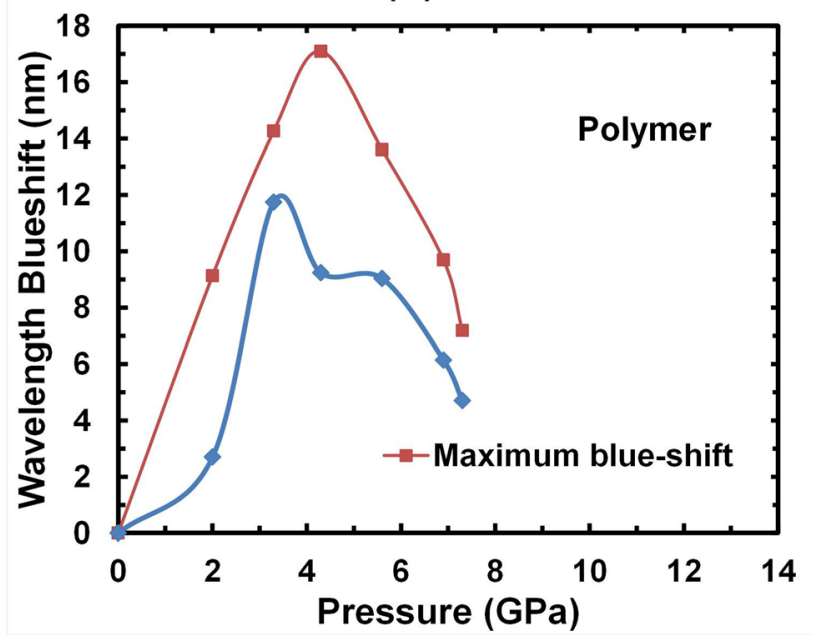

(b)

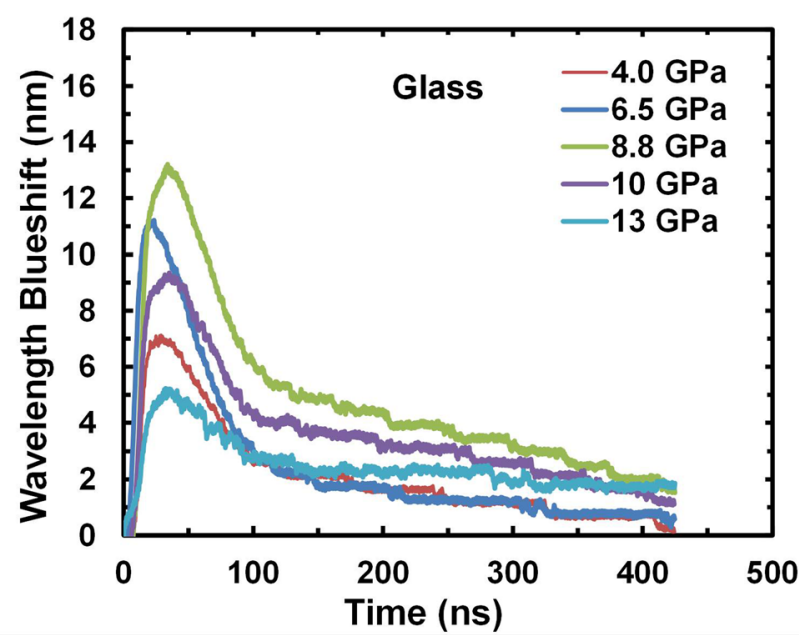

(d)

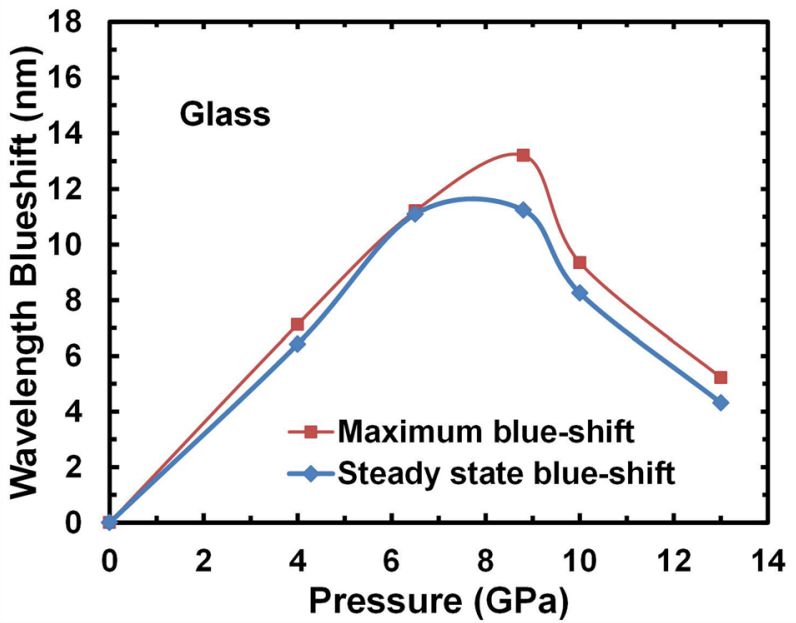

FIG. 4. Spectral blueshifts of QD photoluminescent emission in (a) polymer matrix with 586 nm emitting QDs and (b) glass matrix with 674 nm emitting QDs. The rise and fall of the blueshift are slower in the polymer. The transient blueshift dips in the rising edges for the polymer (in the 4.3-6.9 GPa range) possibly resulting from temporary appearance of small redshifted sideband (c.f. Fig. 2(a)). (c) and (d) Pressure-dependence of blueshifts near the end of steady-state shock stage (at $20 \mathrm{~ns}$ ), depicted in blue, compared to the maximum blueshift, depicted in red. The maximum blueshift was reached much later, at $\sim 60 \mathrm{~ns}$, in the polymer. The blueshift versus pressure relation has a turnover at $\sim 4 \mathrm{GPa}$ in the polymer and $\sim 9 \mathrm{GPa}$ in the glass, possibly due to differences in their volumetric strains.

Similar to experimental results we reported previously ${ }^{13}$ in both glass and polymer, the maximum blueshift does not increase monotonically with increasing shock pressure. Instead there is a turnover pressure where the blueshift reaches a peak and then starts decreasing at $\sim 4 \mathrm{GPa}$ in the polymer and $\sim 9 \mathrm{GPa}$ in the glass. The blueshift pressure turnover under shock is in stark contrast to primarily the monotonic blueshift increase (with no subsequent decrease) observed under static compression reported in Ref. 12.

There are two mechanisms that can lead to the turnover pressure dependence. The first one is due to the coupled temperature and pressure effect. Specifically, the energy gap of the QD is affected by elevated temperature during shock compression, especially after the "ring-up" process in the sample. According to the empirical Varshni equation, the temperature dependence of the energy gap $\left(E_{g}\right)$ of semiconductor crystals can be described as

$$
E_{g}=E_{0}-\alpha \frac{T^{2}}{T+\theta},
$$

where $E_{0}$ is the energy gap at $0 \mathrm{~K}, \theta$ is the Debye temperature (for CdTe $\theta=160 \mathrm{~K}$ ), and $\alpha$ is the temperature sensitivity (for CdTe QD with $d \sim 3 \mathrm{~nm}, \alpha=0.55 \mathrm{meV} / \mathrm{K}$ ). ${ }^{25} \mathrm{Eq}$. (2) suggests that the elevated temperature will decrease the energy gap (redshift). On the other hand, if the QD is under hydrostatic compression, the energy gap is related to the pressure as

$$
E_{g}=E_{g 0}+a_{1} P-a_{2} P^{2},
$$

where $E_{g 0}$ is the energy gap at ambient pressure. For CdTe QD with $d \sim 3.4 \mathrm{~nm}$ at room temperature, $E_{g 0}=2.01 \mathrm{eV}$, the linear factor $a_{l}=46.0 \mathrm{meV} / \mathrm{GPa}$ and nonlinear factor $a_{2}=2.1 \mathrm{meV} / \mathrm{GPa}^{2}$ are obtained from experiment. ${ }^{11}$ Evaluation of Eq. (3) indicates that $E_{g}$ increases monotonically with pressure (blueshift) before phase transformation occurs. ${ }^{11}$ Since temperature in the sample increases along with pressure during shock compression, there will be a competition between the temperature and pressure effects on the energy gap. Though the relation between shock temperature 
and pressure for PVA and glass is not available, a qualitative estimation of the effect can be obtained by using available experimental data of polymethyl methacrylate (PMMA) under shock compression. ${ }^{26}$ Calculated results based on the data of PMMA show a turnover point at $6.0 \mathrm{GPa}$ with a blueshift of $19 \mathrm{~nm}$. The real relation between shock temperature and pressure of PVA and glass should be different from that of PMMA and would lead to a turnover point at smaller or larger pressure level.

The second mechanism is related to non-hydrostatic deformation of the QD. As mentioned in the previous work ${ }^{13}$ and Ref. 12, non-hydrostatic strain states of the QD can result in lower blueshift and non-monotonic dependence on pressure. If the QD is embedded in elastic matrix material softer than the QD and under static uniaxial strain field, according to the solution of the Eshelby's inhomogeneity problem, ${ }^{27}$ the QD will undergo non-hydrostatic deformation with decreased dimension in loading direction and increased transverse dimension. However, when the matrix is under shock compression with pressure beyond its HEL, the QD will experience more hydrostatic loading conditions, especially after the pressure "ring-up" within the QD. So this mechanism will take effect with shock pressure below HEL. In the present study, the HEL for both the PVA and glass matrix is smaller than the examined pressure, so the first mechanism may be expected to play a more dominant role. The coupled temperature and pressure effects also explain the different turnover pressures observed with the QDs dispersed in the polymer $(\sim 4 \mathrm{GPa})$ and in the glass $(\sim 9 \mathrm{GPa})$ matrix.

The time dependence of the blueshift shown in Figures 4(a) and 4(b) reveals interesting features. At a given pressure, the blueshift is larger in the polymer than in the glass matrix. This difference can be attributed in part to different compressibilities and to different QD sizes, since the smaller-size QDs in the polymer have greater quantum confinement, which leads to larger bandgap changes at the same volumetric strain. In the polymer, the blueshift rise times and fall times were much greater than in the glass. Furthermore, in the polymer, the blueshift does not return to its original preshock value within the $450 \mathrm{~ns}$ time window of the experiment, whereas within this time window, the blueshift recovery is nearly complete in the glass. Clearly, there are two recovery processes in the glass samples, one faster and one slower, suggesting two different relaxation stages of volume deformation of QDs embedded in the shocked glass matrix.

An interesting effect is noted in the blueshift for the polymer matrix samples at intermediate $(4.3-6.9 \mathrm{GPa})$ pressures in Figure 4(a). The blueshift first rises, dips, and increases again, all within a few tens of nanoseconds. This effect is clearly real and cannot be attributed to data analysis alone. Note that a smaller, slightly redshifted peak or shoulder appears in Figures 2(a) and 2(c) at around 30 ns. Since the blueshift was computed by the method of moments that finds the average spectral wavelength, the appearance of a secondary peak slightly pulls the average value to longer wavelengths and leads to the transient blueshift dip. As discussed below, the appearance of a new peak or a peak shoulder would also increase the effective peak width determined via the second moment of the spectrum. This blueshift dip prominent in the polymer samples is not observed in the glass samples with larger QDs. The origin of this smaller, secondary peak is unclear based on photoluminescent emission spectra alone. It may, however, be related to fluorescence resonance energy transfer (FRET) ${ }^{28}$ between adjacent QDs of slightly different sizes or, alternatively, a shockinduced QD phase transformation. It has been reported that under hydrostatic conditions there is a zinc blende ( $\mathrm{ZB})$ to cinnabar structure phase transformation in bulk CdTe at $\sim 4 \mathrm{GPa}^{29}$ For nano-sized CdTe QDs, the phase transformation pressure is expected to be size-dependent and higher. A shift of band gap energy to lower levels (longer wavelength) was also reported for $\sim 3 \mathrm{~nm}$ CdTe QDs at $5.8 \mathrm{GPa}$ and attributed to cinnabar to rock salt phase transformation. ${ }^{11}$ The phase transformation pressure of QDs under shock compression conditions is lower than that under hydrostatic compression,$^{30}$ presumably due to the effects of shock induced shear stresses influencing the phase transformation kinetics.

\section{Relative photoluminescent emission intensity decrease}

Figures 5(a) and 5(b) show relative (fractional) intensity decrease as a function of time for the same experiments as in Figure 4 . The corresponding end of shock (20 ns) and maximum intensity decreases are shown in Figures 5(c) and 5(d). Unlike the blueshift exhibiting a pressure turnover, the intensity loss increases monotonically with increasing shock pressure.

The intensity loss has an initial steeper drop followed by a slower recovery. The intensity loss does not show anything comparable to the blueshift dip seen in Figure 4(a) that arises due to the transient appearance of a redshifted photoluminescent emission band in the polymer samples.

The initial intensity drop is slower in the polymer matrix than in the glass matrix, but the much slower intensity recovery is about the same in polymer and in glass. Additionally, the initial intensity loss is slower than the initial blueshift rise, which can be seen by comparing the earliest-time data in Figures 4 and 5 for the polymer and glass matrix samples. These features can be qualitatively understood by considering the different nature of the blueshift and intensity loss processes. The blueshift is an instantaneous electronic response to shock compression, whereas the intensity loss involves shock-induced changes in QD temperature and surface defect states as described in the following paragraph. Because of this reason, the intensity loss may be slower than the spectral shift.

To understand the intensity-loss process, we note that undoped QDs undergo significant intensity loss at temperatures above $100^{\circ} \mathrm{C}$, conditions that the QD composite samples can easily reach during the shock pressures reached in the present work. For example, the estimated shock temperature for PMMA increases from $\sim 50^{\circ} \mathrm{C}$ to $\sim 220^{\circ} \mathrm{C}$ when pressure increases from $2 \mathrm{GPa}$ to $7 \mathrm{GPa}$ pressure. ${ }^{31}$ This loss of photoluminescent emission intensity is most likely closely related to the same process that causes the well-known QD blinking effect. ${ }^{32}$ The prevailing explanation for blinking is 
(a)

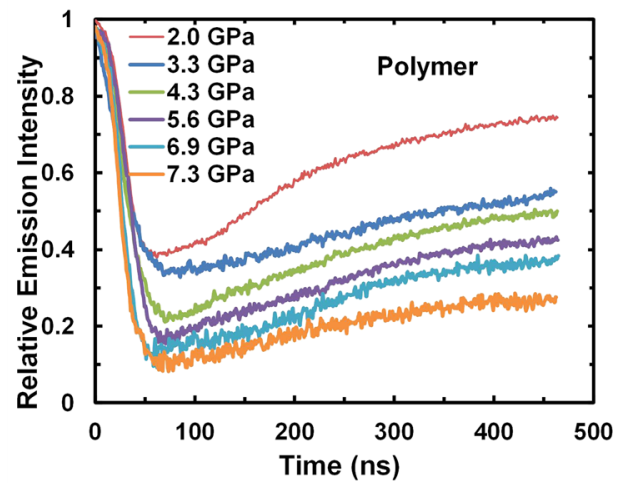

(c)

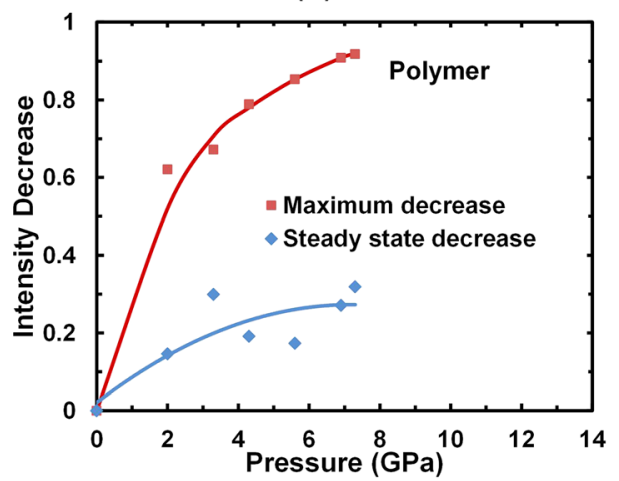

(b)

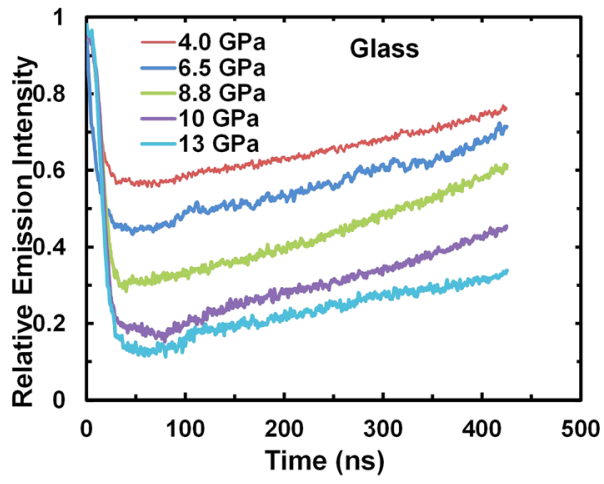

(d)

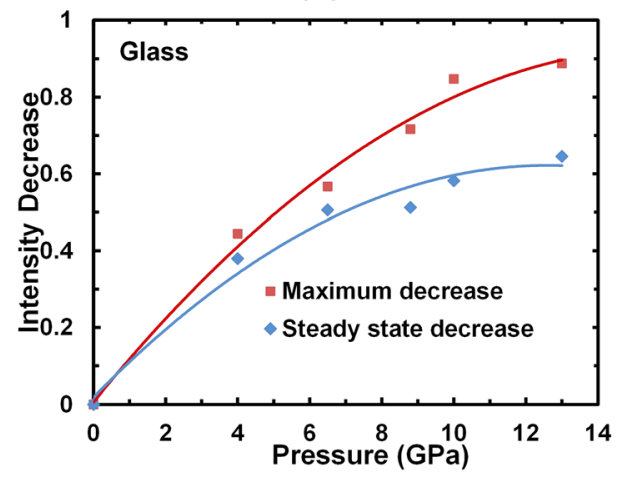

FIG. 5. Time-dependent fractional wavelength-integrated photoluminescent emission intensity loss at different shock pressures for QDs in (a) polymer matrix and (b) glass matrix samples. Plots showing intensity loss versus shock pressure at $20 \mathrm{~ns}$, at end of steady-state shock, and at time of intensity minimum, for (c) polymer and (d) glass. The onset of intensity loss is slower in polymer than in glass but the intensity recovery is similar. The onset of intensity loss is slower than the onset of blueshift (c.f. Fig. 4). that sometimes excited QDs undergo a transfer of either electrons or holes to surface state traps which are dark defect states for which back-transfer is slow. QD blinking is known to become more prominent at higher temperatures. In our shock experiments, some dark defect trap states may be present initially and more may be created by extreme conditions of shock loading such as shearing between the QD and matrix due to uniaxial compression; hence, shock-induced intensity loss may be much more dramatic than the infrequent blinking process.

In the dark state trapping picture, the initial intensity drop is slower in polymer (Figure 5(a)) than in glass (Figure 5 (b)) due to the slower response of the polymer to shockcompression. The initial intensity drop in both the polymer and in the glass is slower than the blueshift because it would take several cycles of laser excitation and QD relaxation to achieve the maximum dark state population, so intensity loss would lag behind blueshift. The recovery of the QD photoluminescent emission intensity, which is slow on the experimental timescale and similar in the polymer and glass, is insensitive to the faster response of the glass to shockcompression because it is controlled by the dark state lifetime. $^{32}$

\section{Shock-induced spectral-width reduction}

Figures 6(a) and 6(b) show the spectral widths of the QD photoluminescent emission peaks obtained from the square-root of the second moment of the spectrum. Since the QD peaks are nearly Gaussian, this quantity is equivalent to the standard deviation. It is striking that shock-compression causes the peak widths to decrease. Despite the high pressure, the anisotropic stress, and the high temperatures, the
QD photoluminescent emission peaks actually sharpen up due to shock-compression. Figures 6(c) and 6(d) show the maximum value of the shock-induced peak narrowing (reduction) plotted as a function of shock pressure.

At a given pressure, the shock-induced peak width reduction is smaller in glass than in polymer. The onset of the peak width reduction is faster in glass but the return to the initial width is about the same in both, the polymer and glass. The peak width stops decreasing at $\sim 4 \mathrm{GPa}$ in the polymer but it keeps increasing up to $12 \mathrm{GPa}$ in the glass matrix.

As shown in Figures 6(a) and 6(b), at the higher shock pressures, the time-dependence of the shock-induced peak width has a non-monotonic behavior. The width first undergoes a temporary increase and then a longer-lasting decrease. The temporary increase is likely due to the appearance of the redshifted sideband responsible for the dips in the shockinduced blueshift seen in Fig. 4(a).

At present, we do not have enough data for a definitive explanation of the shock-induced photoluminescent emission narrowing, but it may be a result of the size distribution of the QDs in the samples, since the emission peak width in QD emitters is ordinarily associated with a size distribution that produces a distribution of emission frequencies. The shockinduced peak narrowing effect occurs in association with a large shock-induced intensity loss, so one possible explanation for the peak narrowing might involve a size-dependence on the surface trapping process.

\section{E. Multiplex probing with dual QDs}

The use of multiple-wavelength emitters to study the behavior of the different particulates that comprise 
(a)

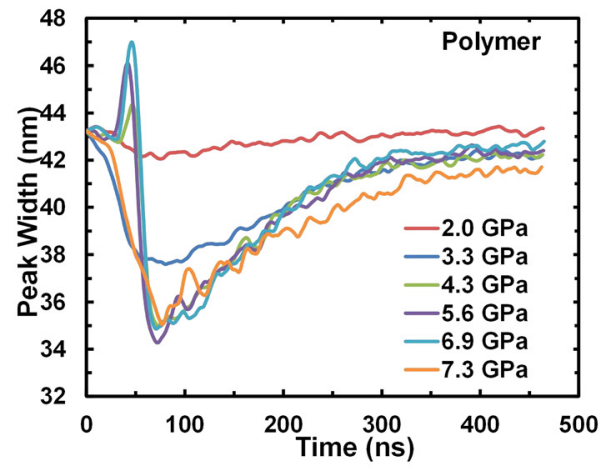

(c)

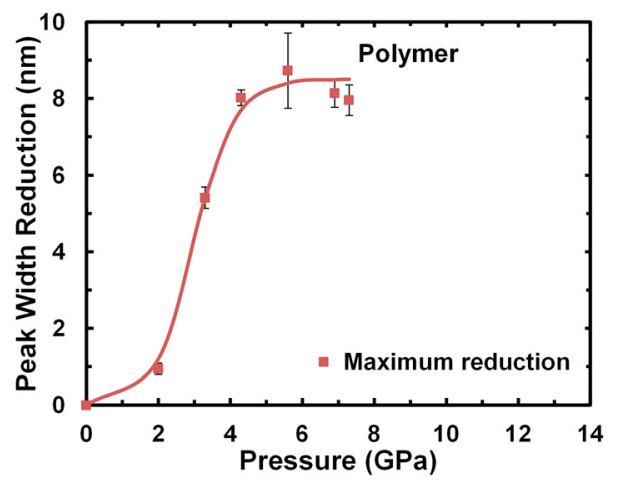

(b)

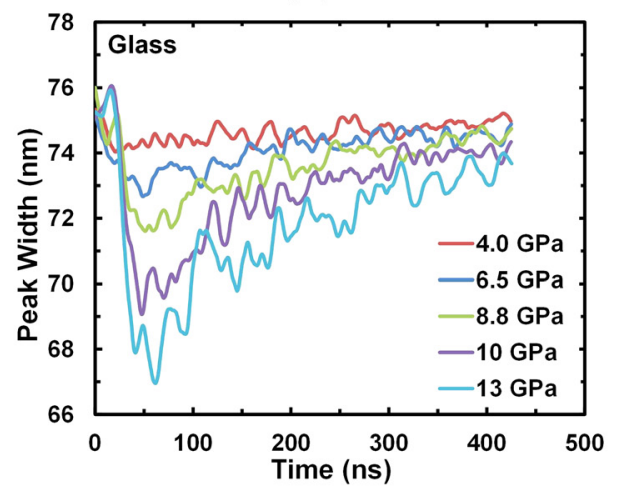

(d)

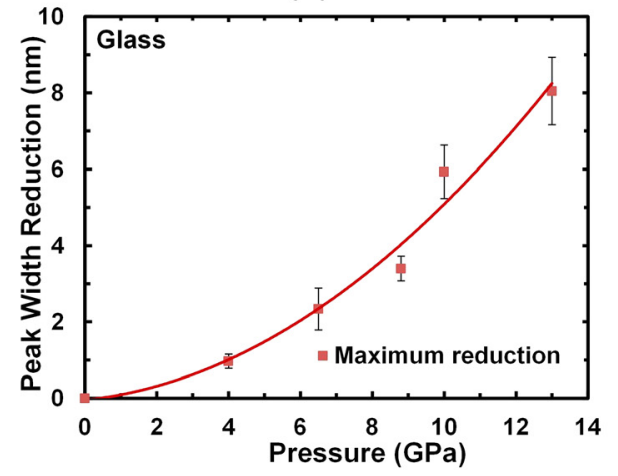

FIG. 6. Shock-induced photoluminescent emission peak-width decrease, expressed as the second moment or standard deviation of the QD emission peaks, and plotted as a function of time in (a) polymer and (b) glass. (c) and (d) Maximum values of peak width reduction for polymer and glass. heterogeneous samples would be possible only if there is minimal energy transfer between the different-color QD probes. To test the feasibility of multiplex probing with dual QDs, we performed an exploratory experiment in which two different kinds of MEA-capped CdTe QDs, emitting either at $582 \mathrm{~nm}$ or $632 \mathrm{~nm}$, were dispersed in a PVA polymer matrix sample. Figure 7(a) shows the time stream photoluminescent emission spectra of a dual-QD sample shock-compressed at a steady-state pressure of 4.2 $\mathrm{GPa}$. It can be seen that both emission peaks are present and well-distinguishable throughout the experiment. The method of moments is not appropriate for a multiplepeak measurement, so the intensity losses, blueshifts, and peak width changes were determined by the more laborious procedure of fitting the spectra to a pair of Gaussian lines, with the results shown in Figures 7(b)-7(d).

It can be seen in Figures 7(b)-7(c) that the two types of QDs have similar time-dependent blueshift and intensity loss behaviors, except that the effects are always greater for the smaller (i.e., the blueshifted) QDs. The spectral peak width dynamics are, however, quite different for the two types of QDs. The larger QDs show a prominent and temporary peak width increase around $45 \mathrm{~ns}$, followed by a small decrease in width, whereas the smaller QDs show a much smaller and temporary peak width increase before settling into a small peak width decrease. The dissimilar peak width responses are most likely related to the different size-dependent phase transition dynamics. These results confirm that the two types of QDs do not exchange significant quantities of electronic energy and behave independently, thereby providing a strong indication for the feasibility of multi-wavelength sensing of shocked materials with embedded QDs.

\section{SUMMARY AND CONCLUDING REMARKS}

We have studied the time-resolved photoluminescence of QDs dispersed in PVA polymer matrix and silicate glass matrix under shock-compression at pressures from 2 to $13 \mathrm{GPa}$. Due to the different compositions, charges, and polarities of these different matrices, a positively-charged QD was needed for the polymer, and a negatively-charged QD for the glass matrix. Otherwise, incompatibility with the matrix would lead to QD aggregation and abnormal photoluminescence behavior. The 586-nm emitting QDs in the polymer were also slightly smaller than the 674-nm emitting QDs in the glass. We also carried out an experiment with QDs of two different sizes (582 and $632 \mathrm{~nm}$ emitting QDs) dispersed in the same PVA polymer matrix.

The arrival of a shock front in the samples causes the QD photoluminescent emission to blueshift, lose intensity and emit in a narrower spectral line. The blueshift arises due to the effects of shock-compression on quantum confinement. Compressing the QDs causes the bandgap to widen and the wavelength to decrease. With shock-compression, the decrease in wavelength (or blueshift) increases with pressure reaching a peak. Above the turnover (peak) pressure, the blueshift decreases, in contrast to the static high pressure blueshift, which increases monotonically with pressure. The blueshift turnover results from the coupled temperature and pressure effect on the band gap of the QDs.

The blueshift is expected to be an instantaneous electronic response to strain. In the limit of instantaneous mechanical response of the embedding matrix medium, the response time of the QDs in terms of their blueshift should be limited by the shock transit time across the sample. That or something close to it appears to occur with the sample 
(a)

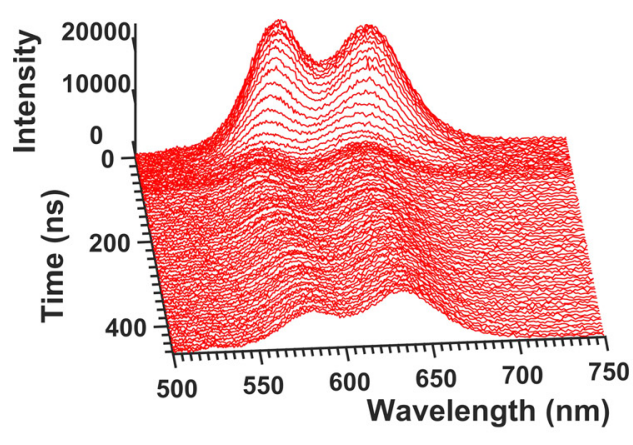

(c)

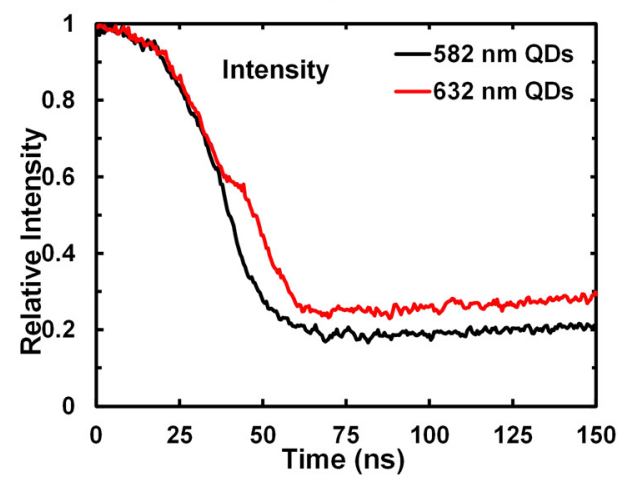

(b)

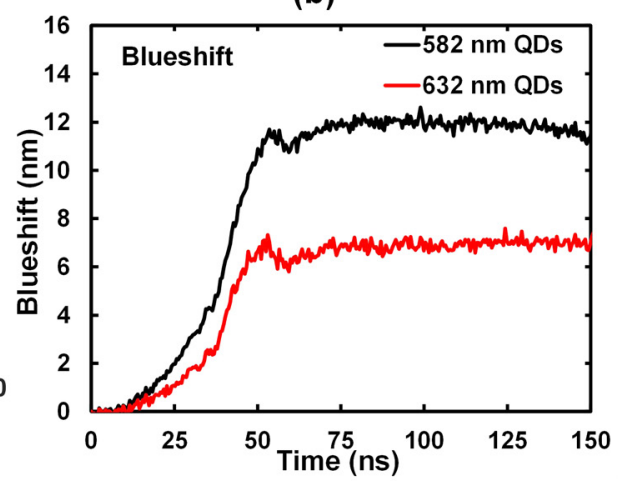

(d)

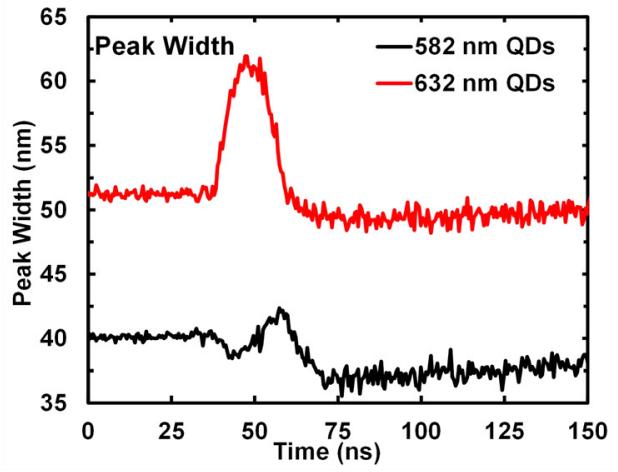

FIG. 7. (a) Time stream photoluminescent emission spectra from dual QDs emitting 582 and 632 wavelengths in the PVA polymer sample shocked at a steady state pressure of $4.2 \mathrm{GPa}$; Plots of (b) wavelength blueshift, (c) emission intensity decrease, and (d) peak width change during the shock pulse as a function of time for the two types of QDs. containing QDs dispersed in the glass matrix. The $30 \mu \mathrm{m}$ sample thickness corresponds to a transit time of $\sim 6 \mathrm{~ns}$, and the blueshift rise time is observed to be of a similar time scale. However, the blueshift response of QDs in the PVA polymer matrix is much slower, with rise times of $\sim 70 \mathrm{~ns}$. It appears that when the mechanical response of the matrix medium containing QDs is sluggish, as in the case of PVA, then the QD photoluminescent emission dynamics are controlled by the response of the surrounding medium.

The intensity loss process was observed to be always slower than the blueshift process. This is because the intensity loss requires both the extreme conditions associated with shock-compression, plus a finite-duration photophysical process. In the case of QDs, we believe that the intensity loss arises from effects similar to those that cause QD blinking, which is usually attributed to electron, or hole, transfer from the QD to dark surface state traps. Once the QDs are subjected to extreme conditions, it takes multiple cycles of excitation and decay to populate a surface trapped state. Even though the intensity loss effect is slower than the blueshift process, intensity losses of up to $90 \%$ are much easier to measure than the observed blueshifts, which in the pressure range studied investigated in the present work, are a fraction of the ambient pressure photoluminescent emission linewidth.

The spectral line narrowing (or peak width reduction) is more difficult to measure accurately than the blueshift and intensity loss. However, it is a useful observation and it is remarkable that the photoluminescent emission narrows under shock-compression. Physical properties such as X-ray diffraction peak widths and optical emission spectra are much more likely to broaden under shock-compression due to the increased temperature and pressure gradients causing changes in lattice spacing. Thus, the peak narrowing (reduction) effect seen with the QDs must result from an interesting dynamical process. It seems possible that the peak reduction effect results from a shock-induced narrowing of the size distribution of the emitting QDs, likely due to the QDs losing intensity by populating dark surface states.

Finally, we have demonstrated the feasibility of multiplex detection of different species in a shock-compressed material, based on an experiment in which two different sized QDs were embedded in the same polymer matrix. Our results show that the necessary conditions for multiplex detection, namely, independent behavior of the different color QDs, can be met.

The dynamic response of QD photoluminescence has been shown in this work to depend on QD composition, size, concentration, and the mechanics of the embedding medium under shock-compression. The diversity of behaviors and the shock-compression responses of the matrix materials complicate the interpretation of photoluminescent emission spectra under shock-compression loading; but, it also offers the high potential for creating and employing diverse types of shock sensors for single and multiplex detection in complex heterogeneous materials, such as particulate media, as demonstrated by the present work.

\section{ACKNOWLEDGMENTS}

The collaborative work described in this paper was funded through two separate projects supported by the Defense Threat Reduction Agency (DTRA) Grant No. HDTRA1-12-1-0052 at Georgia Tech and HDTRA1-12-10011 at the University of Illinois at Urbana Champaign. The 
authors gratefully acknowledge the encouragement and support provided by the program monitors Dr. Suhithi Peiris and Dr. Douglas (Allen) Dalton. The work was also supported in part by the U.S. Air Force Office of Scientific Research under Award No. FA9550-15-1-0499, at Georgia Tech, and FA9550-16-1-0042 at the University of Illinois at Urbana Champaign.

${ }^{1}$ J. W. Forbes, Shock Wave Compression of Condensed Matter: A Primer (Springer, New York, NY, 2012).

${ }^{2}$ R. A. Graham, F. W. Neilson, and W. B. Bennedick, "Piezoelectric current from shock-loaded quartz-A submicrosecond stress gauge," J. Appl. Phys. 36(5), 1775-1783 (1965).

${ }^{3}$ Z. Rosenberg and Y. Partom, "Longitudinal dynamic stress measurements with in-material piezoresistive gauges," J. Appl. Phys. 58(5), 1814-1818 (1985).

${ }^{4}$ T. J. Vogler, W. M. Trott, W. D. Reinhart, C. S. Alexander, M. D. Furnish, M. D. Knudson, and L. C. Chhabildas, "Using the line-VISAR to study multi-dimensional and mesoscale impact phenomena," Int. J. Impact Eng. 35, 1844-1852 (2008).

${ }^{5}$ D. C. Swift, J. G. Niemczura, D. L. Paisley, R. P. Johnson, S. N. Luo, and T. E. Tierney IV, "Laser-launched flyer plates for shock physics experiments," Rev. Sci. Instrum. 76, 093907 (2005).

${ }^{6}$ R. J. Trainor, J. W. Shaner, J. M. Auerbach, and N. C. Holmes, "Ultrahigh-pressure laser-driven shock-wave experiments in aluminum," Phys. Rev. Lett. 42, 1154 (1979).

${ }^{7}$ W. C. Chan, "Quantum dot bioconjugates for ultrasensitive nonisotropic detection," Science 281, 2016 (1998).

${ }^{8} \mathrm{M}$. Narayanan and A. J. Peter, "Pressure and temperature induced nonlinear optical properties in a narrow band gap quantum dot," Quantum Matter 1, 53 (2012).

${ }^{9}$ H. G. Drickamer, C. W. Frank, and C. P. Slichter, "Optical versus thermal transitions in solids at high pressure," Proc. Natl. Acad. Sci. U.S.A. 69, 933 (1972).

${ }^{10}$ E. Pedrueza, A. Segura, R. Abargues, J. B. Bailach, J. C. Chervin, and J. P. Martínez-Pastor, "The effect of quantum size confinement on the optical properties of PbSe nanocrystals as a function of temperature and hydrostatic pressure," Nanotechnology 24, 205701 (2013).

${ }^{11}$ Y. Lin, "Water-soluble CdTe nanocrystals under high pressure," Proc. SPIE 9373, 93730L (2015).

${ }^{12}$ F. Wu, J. M. Zaug, C. E. Young, and J. Z. Zhang, "Pressure-induced phase transition in thiol-capped CdTe nanoparticles," J. Nanosci. Nanotechnol. 8, 6528 (2008).

${ }^{13}$ P. Xiao, Z. Kang, A. A. Bansihev, J. Breidenich, D. A. Scripka, J. Christensen, C. J. Summers, D. D. Dlott, N. N. Thadhani, and M. Zhou, "Laser-excited optical emission response of CdTe quantum dot/polymer nanocomposite under shock compression," Appl. Phys. Lett. 108, 011908 (2016).

${ }^{14} \mathrm{C}$. Neel and N. N. Thadhani, "Shock and release wave speed of an alumina epoxy composite," J. Appl. Phys. 106(4) 0461051 (2009).
${ }^{15}$ J. Cagnoux, P. Chartagnac, P. Hereil, and M. Perez, "Lagrangian analysis. Modern tool of the dynamics of solids," Ann. Phys. Fr. 12, 451-524 (1987).

${ }^{16} \mathrm{Z}$. Kang et al., "CdTe quantum dots and polymer nanocomposites for $\mathrm{x}$ ray scintillation," Appl. Phys. Lett. 98, 181914 (2011).

${ }^{17}$ H. Zhang, L. Wang, H. Xiong, L. Hu, B. Yang, and W. Li, "Hydrothermal synthesis for high-quality CdTe nanocrystals," Adv. Mater. 15, 1712 (2003).

${ }^{18}$ K. E. Brown, Y. Fu, W. L. Shaw, and D. D. Dlott, "Time-resolved emission of dye probes in a shock-compressed polymer," J. Appl. Phys. 112, 103508 (2012).

${ }^{19}$ B. J. Jensen, D. B. Holtkamp, P. A. Rigg, and D. H. Dolan, "Accuracy limits and window corrections for photon Doppler velocimetry," J. Appl. Phys. 101, 013523 (2007).

${ }^{20}$ A. D. Curtis, A. A. Banishev, W. L. Shaw, and D. D. Dlott, "Laser-driven flyer plates for shock compression science: Launch and target impact probed by photon Doppler velocimetry," Rev. Sci. Instrum. 85, 043908 (2014).

${ }^{21}$ S. Chaurasia, P. Leshma, J. Pasley, S. Tripathi, and M. Kumar, "Shock pressure multiplication in layered PVA + Al targets driven by a highpower laser pulse," Curr. Sci. 103, 1447 (2012).

${ }^{22}$ C. S. Alexander, L. C. Chhabildas, and D. W. Templeton, "The Hugoniot elastic limit of soda-lime glass," AIP Conf. Proc. 955, 733 (2007).

${ }^{23}$ A. A. Banishev, W. L. Shaw, and D. D. Dlott, "Dynamics of polymer response to nanosecond shock compression," Appl. Phys. Lett. 104, 101914 (2014).

${ }^{24}$ J. Wackerle, "Shock-wave compression of quartz," J. Appl. Phys. 33, 922-937 (1962).

${ }^{25} \mathrm{~N}$. Vyhnan and Y. Khalavka, "Size-dependent temperature sensitivity of photoluminescence peak position of CdTe quantum dots," Luminescence 29, 952-954 (2014).

${ }^{26}$ S. A. Bordzilovsky, M. S. Voronin, S. M. Karakhanov, and L. A. Merzhievsky, "Shock-compression temperature of polymeric materials," Dokl. Phys. 59, 176-180 (2014).

${ }^{27}$ J. W. Ju and L. Z. Sun, "Effective elastoplastic behavior of metal matrix composites containing randomly located aligned spheroidal inhomogeneities. Part I: Micromechanics-based formulation,” Int. J. Solids Struct. 38, 183-201 (2001).

${ }^{28} \mathrm{Y}$. Li, Q. Ma, X. Wang, and X. Su, "Fluorescence resonance energy transfer between two quantum dots with immunocomplexes of antigen and antibody as a bridge," Luminescence 22, 60-66 (2007).

${ }^{29}$ M. G. Brik, P. Qach, G. Karczewski, T. Wojtowicz, A. Kaminska, and A. Suchocki, "Theoretical studies of the pressure-induced zinc-blende to cinnabar phase transition in CdTe and thermodynamical properties of each phase," Matl. Chem. Phys. 140, 216-221 (2013).

${ }^{30} \mathrm{~J}$. S. Wittenberg, M. G. Merkle, and A. P. Alivisatos, "Wurtzite to rocksalt phase transformation of cadmium selenide nanocrystals via laser-induced shockwaves: Transition from single to multiple nucleation," Phys. Rev. Lett. 103, 125701 (2009).

${ }^{31}$ W.-L. Liu, W. P. Bassett, J. M. Christensen, and D. D. Dlott, "Emission lifetimes of a fluorescent dye under shock compression," J. Phys. Chem. A 119, 10910-10916 (2015).

${ }^{32} \mathrm{~S}$. Hohng and T. Ha, "Near-complete suppression of quantum dot blinking in ambient conditions," J. Am. Chem. Soc. 126, 1324-1325 (2004). 